\title{
p90RSK Activation Promotes Epithelial-Mesenchymal Transition in Cisplatin-Treated Triple-Negative Breast Cancer Cells
}

\author{
Yujin Jin and Kyung-Sun Heo* \\ College of Pharmacy and Institute of Drug Research and Development, Chungnam National University, \\ Daejeon, South Korea
}

\section{Corresponding}

Kyung-Sun Heo

College of Pharmacy and Institute of Drug

Research and Development, Chungnam

National University, Daejeon, South Korea

Phone : +82-42-821-5927

Fax : +82-42-821-8925

E-mail : kheo@cnu.ac.kr

Received : December 9, 2019

Revised : December 16, 2019

Accepted : December 17, 2019

No potential conflict of interest relevant to this article was reported.

Copyright (C) 2019 Journal of Bacteriology and Virology

CThis is an Open Access article distributed under the terms of the Creative Commons Attribution Non-Commercial License (http://creativecommons.org/ license/by-nc/3.0/) p90 ribosomal S6 kinase (p90RSK), one of the downstream effectors in ERK1/2 pathways, shows high expression in human breast cancer tissues. However, its role in breast cancer development and drug resistance is not fully understood. Here, we demonstrate that Cis-DDP treatment failed to increase cytotoxicity in MDA-MB-231 cells compared to MCF-7 cells and p90RSK activation was involved in Cis-DDP-resistance to MDA-MB-231 cells. In the study, we found that inhibition of p90RSK expression or activation using a small interfering RNA (siRNA) or dominant-negative kinase mutant (DN-p90RSK) plasmid overexpression increased Cis-DDP-induced cytotoxicity of MDA-MB-231 cells, respectively. Mechanistically, we found that Cis-DDP resistance was associated with up-regulation of epithelial growth factor (EGF) expression and EGF treatment induced cancer survival signaling pathway including activation of ERK1/2, p90RSK, and Akt. We also examined the expression of epithelial-mesenchymal transition (EMT)-associated proteins using a reverse transition-quantitative PCR analysis. Cis-DDP treatment induced EMT by increasing the expression levels of N-cadherin, Snail, and Twist, while decreasing the expression levels of E-cadherin. Furthermore, we examined the epithelial marker, Zonula occludens-1 (ZO-1) using immunofluorescence analysis and found that Cis-DDP-inhibited ZO-1 expression was recovered by p90RSK deactivated condition. Therefore, we conclude that Cis-DDP resistance is involved in EMT via regulating the EGF-mediated p90RSK signaling pathway in MDA-MB-231 cells.

Key Words: Cisplatin, Epithelial-mesenchymal transition, p90RSK, Triple-negative breast cancer cells, Breast Cancer, Cisplatin, Epithelial-mesenchymal transition, p90RSK

\section{INTRODUCTION}

Cisplatin (Cis-DDP) is an anti-cancer drug that is classified as an alkylating agent, which is one of the most widely used anti-cancer drugs (1-3). Although it is applicable to many types of cancer, including cervical, lung, bladder, and breast cancer, its use is now limited because of drug resistance.

The hormone receptors for breast cancer cell line consist of estrogen receptor (ER), progesterone receptor (PR), and human epithelial growth factor 2 (HER2), and divided into ER+, ER-, HER2+, and triple negative breast cancer (TNBC) according to the expression of each receptor (4). Of these cell types, the TNBC is limited because TNBC is insensitive to most hormonal and therapeutic agents (5). 
Therefore, TNBC exhibits high recurrence and metastasis with poor prognosis (5). Various clinical trials including therapies of cisplatin (Cis-DDP) alone or combination with other agents were tested $(6,7)$, but Cis-DDP resistance to breast cancer is not fully understood.

It has been demonstrated that overexpressed level of p90 ribosomal protein S6 kinase (p90RSK), which is a downstream substrate of ERK1/2 signaling, was shown in invasive breast cancer tissue (8). p90RSK contains two functional kinase domains, an N-terminal kinase domain (NTKD) and a C-terminal kinase domain (CTKD) connected by a linker domain $(9,10)$. Once ERK $1 / 2$ is activated by various stimuli, such as carcinogens and growth factors, it causes phosphorylation of Thr577 in the CTKD of p90RSK and sequential phosphorylation of Thr365 and Ser386 in the linker region (11). These phosphorylations are associated with the activation of p90RSK substrates, including transcription factors, c-Fos, and Myt1 (12, 13).

Epithelial-mesenchymal transition (EMT) occurs due to the loss of epithelial markers, E-cadherin and Zonula occludens-1 (ZO-1), via many growth factor signaling pathways (14). It has been reported that an EMT transcription factor, Twist correlates with Ras-activated mitogen-activated protein kinase (MAPK), which is one of the signaling pathways involved in the promotion of breast cancer cell invasion (15). In addition, various transcription factors are related to EMT and cell invasion, and Slug, Snail, and Twist are transcription factors that have been reported to regulate the expression of tumor suppressor such as E-cadherin (16).

In this study, we investigated the role of p90RSK, the downstream effector of the MAPK pathway in the proliferation of Cis-DDP-treated breast cancer cells. We discovered that p90RSK activation was involved in Cis-DDP-resistance to MDAMB-231 cells. The Cis-DDP resistance was associated with up-regulation of epithelial growth factor (EGF) expression and EMT-associated proteins including N-cadherin, Snail, and Twist. Therefore, our study aimed to investigate whether the regulation of p90RSK activity is a critical therapeutic target for increasing Cis-DDP sensitivity in patients with TNBC.

\section{MATERIALS AND METHODS}

\section{Cell lines and Reagents}

Human mammary carcinoma cell lines MDA-MB-231 (HTB-26 ${ }^{\mathrm{TM}}$ ) and MCF-7 (AHTB-22 ${ }^{\mathrm{TM}}$ ) were obtained from the American Type Culture Collection (Manassas, VA, USA). MDA-MB-231 cells express none of hormone receptors, so they are classified as TNBC and MCF-7 cells express ER. Rabbit anti-phospho-Akt Ser473, rabbit anti-Akt, rabbit anti-phospho-p90RSK Ser380, rabbit anti-phospho-ERK1/2, and rabbit anti-ERK1/2 antibodies were purchased from Cell Signaling Technology, Inc. (Danvers, MA, USA). Immobilon-P (\#IPVH00010) were purchased from Merck Millipore (Burlington, MA, USA). 10X Phosphate buffered saline (PBS, \#EBA-1105) and reveres transcription 5X master mix (\#EBT-1511) were purchased from ELPIS-BIOTECH (Daejeon, South Korea). Tri-RNA reagent (\#FATRR-001) was purchased from Favorgen (Pingtung, China). Cis-DDP (\#C2210000), 2-mercaptoethanol (\#M6250), and skim milk (\#70166) were purchased from Sigma-Aldrich (St. Louis, MO, USA). Clarity western ECL substrate (\#170-5061) and iQ SYBR green supermix (\#170-8882AP) were purchased for BIO-RAD (Hercules, CA, USA). EzReprobe (\#WSE-7240) and EzRIPA Lysis kit (\#WSE-7240) were purchased for ATTO corporation (Tokyo, Japan). Dulbecco's modified Eagle's medium (DMEM, \#11963-092), Fetal bovine serum (FBS, \#26140-079), Penicillin streptomycin (P/S, \#1570-063) and 0.25\% Trypsin/EDTA (\#25200-072) were purchased from Gibco (ThermoFisher Scientific; Waltham, MA, USA). 3-(4,5-dimethylthiazole-2-yl)-2 and Lipofectamine 3000 (\#L3000-008) were purchased from Invitrogen (ThermoFisher Scientific). FMK was purchased from Axon Medchem (\#Axon 1848, Reston, VA, USA). 


\section{MTT analysis}

Cell viability was determined using 3-(4,5-dimethylthiazole-2-yl)-2, 5-diphenyltetrazolium bromide assay (MTT, \#M6494, Sigma-Aldrich) assay. For the assay, cells were seeded in 96-well at a density of $1 \times 10^{4}$ cells per well. After $24 \mathrm{~h}$ of stabilization, the cells were treated with various dose of Cis-DDP for $24 \mathrm{~h}$. Cells were treated with MTT (12 mM) in FBS-free DMEM media and incubated for $2 \mathrm{~h}$ at $37^{\circ} \mathrm{C}$ and $5 \% \mathrm{CO}_{2}$. The medium was the removed and the formazan precipitate was extracted in DMSO. The absorbance was measured at $540 \mathrm{~nm}$ on a microplate reader (TECAN, Männedorf, Switzerland).

\section{Flow cytometry analysis}

The Flow cytometry analysis was used to measure cell viability using a Muse count \& viability assay kit (\#MCH100102, Merck Millipore) according to the manufacture's protocol. Briefly, MDA-MB-231 and MCF-7 cells were seeded in 6-well plate at a density of $1 \times 10^{6}$ cells/well. For cell proliferation experiment, cells were harvested after 24-36 h treated with Cis-DDP. Cells were directly stained by reagent provided by kit and the cell viability analysis was performed using a Muse cell analyzer (EMD Millipore) and data were analyzed using a MUSE 1.5 analysis program.

\section{Western blot analysis}

Western blotting analysis were performed as described previously (17). Briefly, total protein samples were separated by SDS-PAGE and transferred to nitrocellulose membranes. Western blotting was performed using an each corresponding specific antibody. Polyclonal mouse anti- $\alpha$-tubulin (Sigma-Aldrich) was used as an internal control.

\section{Cell transfection}

Plasmids including pcDNA and dominant negative (DN)-p90RSK were transfected with EndoFectin ${ }^{\mathrm{TM}}$ Max transfection reagent (\#EF014, GeneCopoeia, Rockville, VA, USA) according to the manufacture's protocol. Rat RSK1 (NM031107) was mutated to K94A/K447A to create a kinase dead protein (DN-p90RSK1) with the QuickChange II site-directed mutagenesis kit (\#200521, Agilent, Santa Clara, CA, USA) as described previously (17).

\section{Real-Time Polymerase Chain Reaction assay}

The quantitative real-time polymerase chain reaction ( $q R T-P C R$ ) assay was used to analyze the mRNA expression of E-cadherin, N-cadherin, Snail, and Twist as described previously (17). The relative gene expression was calculated using the $2^{-\Delta c t}$ method, and GAPDH was used for normalization. All primer sequences used in qRT-PCR experiments are listed in Table 1.

Table 1. Primer sequence of genes

\begin{tabular}{ccc}
\hline Genes & Forward primer sequence & Reverse primer sequence \\
\hline Snail & CCCCAATCGGAAGCCTACT & GCTGGAAGGTAAACTCTGGATTAGA \\
Twist & GGAGTCCGCAGTCTTACGAG & TCTGGAGGACCTGGTAGAGG \\
E-Cadherin & AATTCCTGCCATTCTGGGGA & TCTTCTCCGCCTCCTTCTTC \\
N-cadherin & TGAGCCTGAAGCCAACCTTA & AGGTCCCCTGGAGTTTCTG \\
GAPDH & GCACCGTCAAGGGCTGAGAAC & TGGTGAAGACGCCAGTGGA \\
\hline
\end{tabular}

\section{p90RSK knockdown}

To knockdown p90RSK in vitro, specific short interference RNA (siRNA) was used (\#SC-29475, Santa Cruz Biotechnology, Santa Cruz, CA, USA). A siRNA that does not match any known human coding CDNA was used as a negative control for 
silencing (Scramble, sc-37007, Santa Cruz Biotechnology). Transfections were performed using Lipofectamine 3000 (Invitrogen) according to the manufacturer's instructions.

\section{Immunofluorescence staining}

Immunofluorescence assay was performed as described previously (17). Briefly, cells were fixed with $4 \%$ paraformaldehyde for 15 min at RT and permeabilized with $0.1 \%$ Triton X-100 for 20 min. Cells were incubated with the primary antibodies (ZO-1 1:500) overnight at $4^{\circ} \mathrm{C}$ followed by incubation with anti-rabbit secondary antibodies (Invitrogen) conjugated with Alexa 488 at a dilution of 1:400 at RT for $1 \mathrm{~h}$. Cell nuclei were counterstained with 40, 6-diamidino-2-phenylindol (DAPI) for $5 \mathrm{~min}$. Slides were mounted with prolong gold antifade mount reagent (\#P36930, Invitrogen) and examined with a laser scanning confocal spectral microscope (K1-Fluo, Nanoscope systems, Daejeon, South Korea). Representative images were automatically taken using a SPOT digital camera.

\section{Statistical analysis}

Statistical analysis was performed using GraphPad Prism 5 (version 5.02, GraphPad Software Inc., San Diego, CA, USA). One-way analysis of variance (ANOVA) followed by a Bonferroni multiple comparison was performed. A $p$ value $<0.05$ was considered significant. All experiments were expressed as the mean \pm SEM and were performed independently at least 3 times.

\section{RESULTS}

\section{Effect of Cis-DDP on cell viability of breast cancer cells}

We evaluated Cis-DDP resistance on cell viability in MDA-MB-231 and MCF-7 cells. Cell viability tests were performed by flow cytometry using Muse count \& cell viability kit. The serum starved breast cancer cells were treated with various concentrations of Cis-DDP $(1,5,10$, and $20 \mu \mathrm{g} / \mathrm{mL})$ for $36 \mathrm{hr}$. Cis-DDP treatment significantly induced cytotoxicity in MCF-7 cells, whereas MDA-MB-231 exhibited the resistance to cell viability after Cis-DDP treatment (Fig. 1).

\section{A MDA-MB-231}
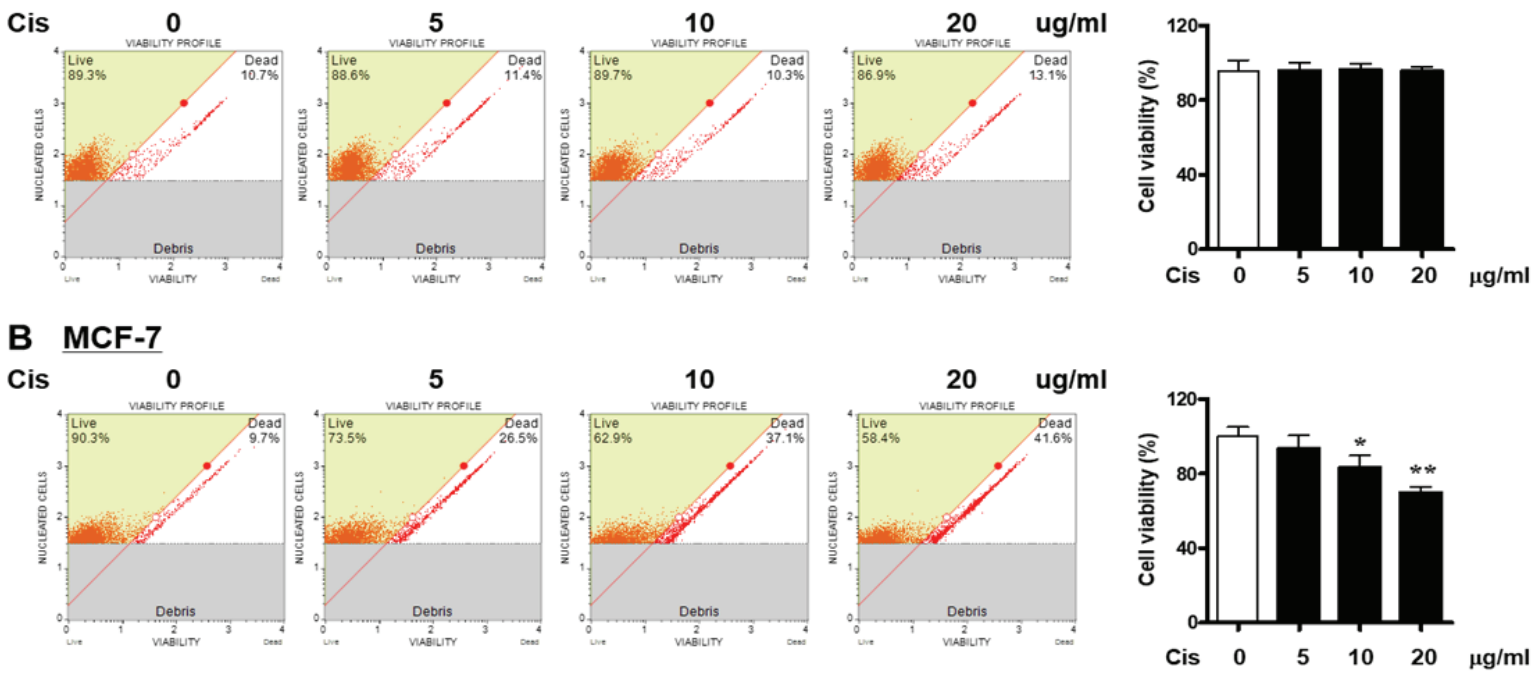

Fig. 1. Effect of Cis-DDP on cell viability of breast cancer cells MDA-MB-231 and MCF-7 cells were treated with indicated various concentrations of Cis-DDP for $36 \mathrm{~h}$. cell viability was assessed using the Muse cell count \& viability. Data are the mean \pm SEM of experiments in triplicate $(n=3) .{ }^{*} P<0.05,{ }^{* *} P<0.01$ compared with 0 sample. 


\section{Involvement of p90RSK activation in cell viability of MDA-MB-231 cells}

Previous report demonstrated that overexpression of p90RSK was shown in breast cancer tissues (8). To identify the p90RSK-mediated signaling pathway associated with Cis-DDP resistance to MDA-MB-231 cells, cells were transfected with siRNA of control (si-Cont) or p90RSK (si-RSK1) for $48 \mathrm{hr}$. As shown in Fig. 2A, Cis-DDP treatment increased activation of p90RSK, whereas depletion of p90RSK inhibited Cis-DDP induced both p90RSK activation and expression. In addition, si-RSK1 transfected cells significantly sensitized cells to Cis-DDP induced cytotoxicity compared with si-Cont transfected cells (Fig. 2B). Next experiment was confirmed the effect of p90RSK activation on Cis-DDP induced cytotoxicity using transfected cells with plasmid of wild type (WT)-p90RSK and DN-p90RSK. WT-p90RSK induced Cis-DDP resistance to cytotoxicity of MDA-MB-231 cells, whereas, DN-p90RSK sensitized the cells to Cis-DDP induced cytotoxicity via deactivation of p90RSK (Fig. 2C). Together, we demonstrated the important role of p90RSK activation in Cis-DDP resistance in TNBC cytotoxicity.
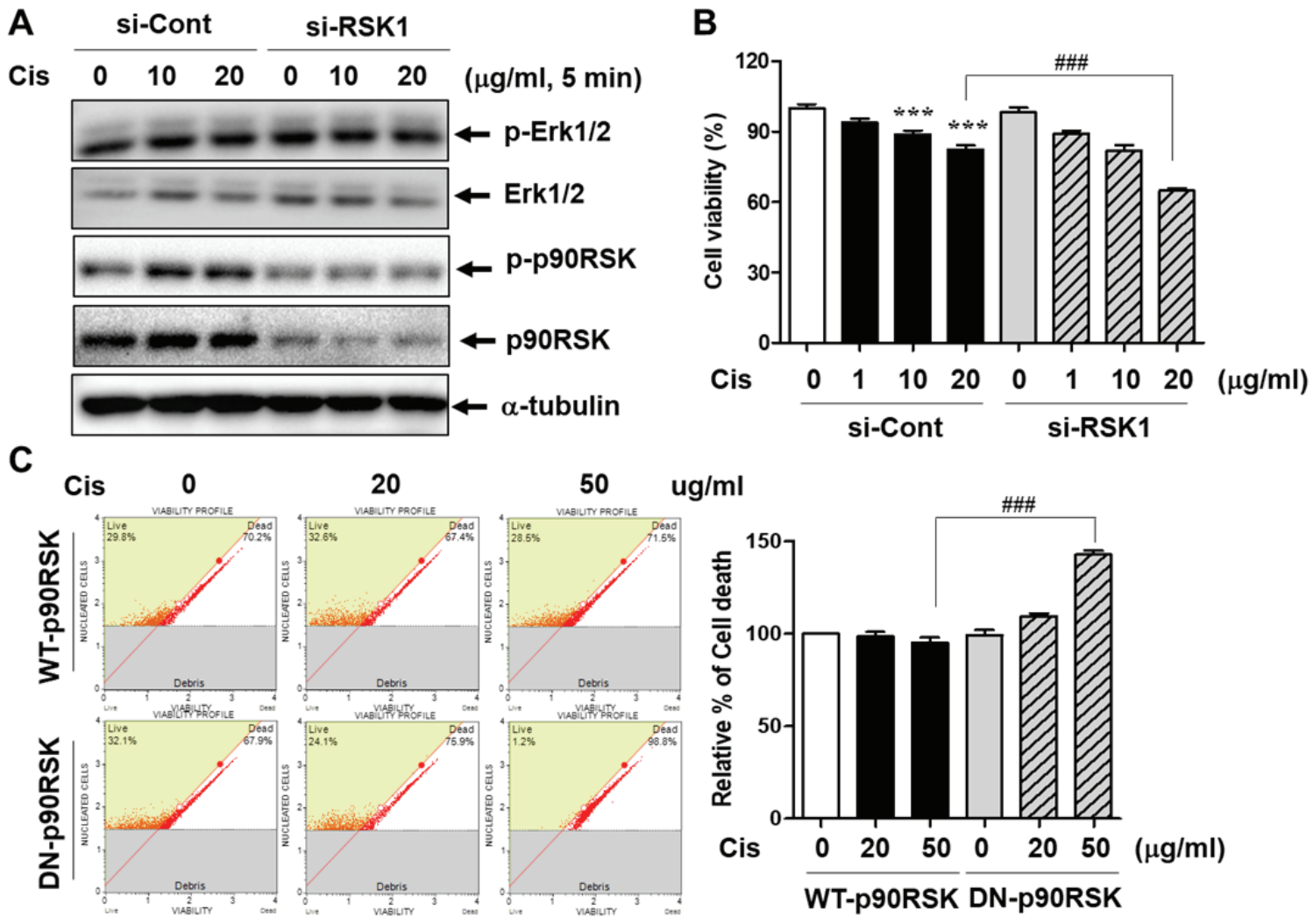

Fig. 2. Involvement of p90RSK activation in cell viability of MDA-MB-231 cells (A) MDA-MB-231 cells transfected with si-Cont or si-RSK1 for $48 \mathrm{~h}$ followed by treatment with the indicated concentrations of Cis-DDP for $5 \mathrm{~min}$. Whole cell lysates were subjected to western blot analysis against the indicated antibodies. (B) MDA-MB-231 cells were transfected with si-Cont or si-RSK1 for $18 \mathrm{~h}$ followed by treatment with the indicated concentrations of Cis-DDP for $24 \mathrm{~h}$ and cell viability was determined by an MTT assay. (C) MDA-MB-231 were transfected with WT-p90RSK or DN-p90RSK for $18 \mathrm{~h}$ followed by treatment with the indicated concentrations of Cis-DDP for $24 \mathrm{~h}$ and cell viability was determined by flow cytometry. The data are presented as means \pm SEM $(n=3) .{ }^{* * \star} P<0.001$ compared with 0 sample; ${ }^{\# \#} P<0.001$ compared with each control in the siCont or WT-p90RSK, respectively.

\section{Effect of p90RSK activation on hEGF-induced cell survival pathways}

It has been known that EGF signaling pathway was related to cancer cell survival and drug resistance in breast cancer cells (18). Therefore, we examined whether Cis-DDP resistance was associated with up-regulation of EGF expression in MDA-MB-231 cells using qRT-PCR analysis. As shown in Fig. 3A, Cis-DDP increased mRNA expression level of EGF in a 
dose-dependent manner. Next, we investigated if p90RSK activation is involved in EGF-mediated cancer cell survival signaling pathways using a FMK, which is a p90RSK specific inhibitor. Recombinant protein of human EGF (hEGF) treatment induced phosphorylations of ERK1/2, p90RSK, and Akt at $5 \mathrm{~min}$, and these maximum phosphorylations were seen at 60 min, respectively (Fig. 3B). Interestingly, FMK pretreatment strongly abolished hEGF-induced phosphorylation of p90RSK, but not phosphorylations of ERK1/2 and Akt. It suggests that FMK specifically inhibited only p90RSK activation and Cis-DDP resistance to TNBC is associated with EGF-induced p90RSK activation.

A

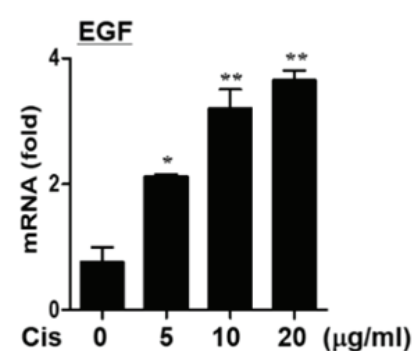

B

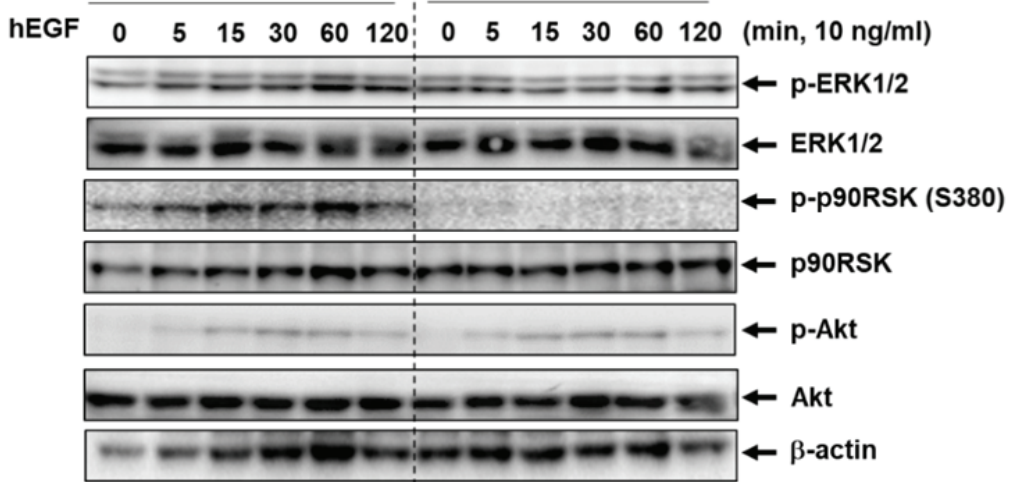

Fig. 3. Effect of p90RSK activation on hEGF-induced cell survival pathways (A) MDA-MB-231 cells were stimulated with Cis-DDP $6 \mathrm{~h}$ and mRNA expression level was determined by qRT-PCR. (B) MDA-MB-231 cells were pretreated with $10 \mathrm{nM}$ of FMK for $1 \mathrm{~h}$ followed by treatment of $10 \mathrm{ng} / \mathrm{ml}$ hEGF for selected time intervals (0 120 min). Whole cell lysates were subjected to western blot analysis against the indicated antibodies. The data are presented as means $\pm \mathrm{SEM}(n=3)$. ${ }^{* *} P\langle 0.01$ compared with 0 sample.

\section{Effect of p90RSK activation on EMT markers}

It has been reported that anti-cancer drug resistance was related to EMT $(3,19)$. We examined the role of p90RSK activation on expression of EMT-associated proteins using a qRT-PCR analysis. MDA-MB-231 cells were transfected si-Cont or si-RSK for $48 \mathrm{~h}$ followed by treatment with 0,10 , and $20 \mu \mathrm{g} / \mathrm{ml}$ of Cis-DDP for $6 \mathrm{~h}$. As shown in Fig. 4A-D, Cis-DDP treatment induced EMT by increasing the mRNA expression levels of N-cadherin, Snail, and Twist, while decreasing the expression levels of E-cadherin. The condition of depletion of p90RSK resulted in decreased levels of Cis-DDP-induced EMT markers and increased level of Cis-DDP-inhibited E-cadherin. We further examined epithelial marker, ZO-1 using immunofluorescence analysis and found that Cis-DDP-inhibited ZO-1 expression was recovered by p90RSK deactivated condition (Fig. 4E). Together, our results indicats that p90RSK activation is involved in the regulation of EMT for causing Cis-DDP resistance in TNBC.

\section{Discussion}

The purpose of this study was to determine the role of p90RSK activation and its underlying signaling pathway on Cis-DDP drug resistance in breast cancer cells. Our study demonstrated that p90RSK kinase activation is involved in Cis-DDP-mediated cell viability and EMT.

Previous report demonstrated that overexpression of p90RSK was shown in breast cancer tissues (8). In the report, they developed an inhibitor of p90RSK, SL0101, and investigated effect of the inhibitor on TNBC treatment. The SL0101 binds to an N-terminal kinase domain of p90RSK and inhibits p90RSK kinase activity. Although they suggested the inhibitor as a new drug of TNBC treatment, effect of the inhibitor on specific signaling pathways were insufficient. 
A

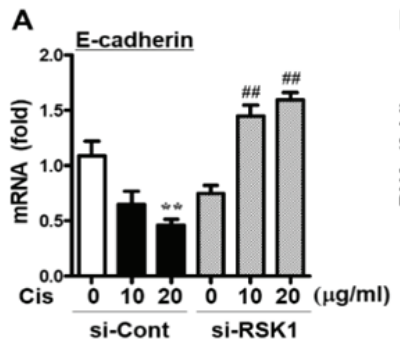

B

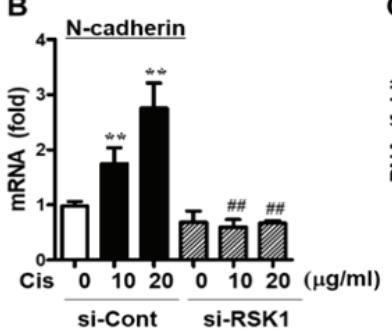

C

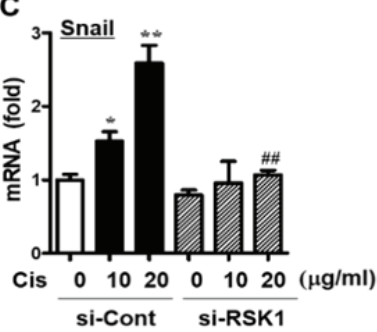

FMK

E

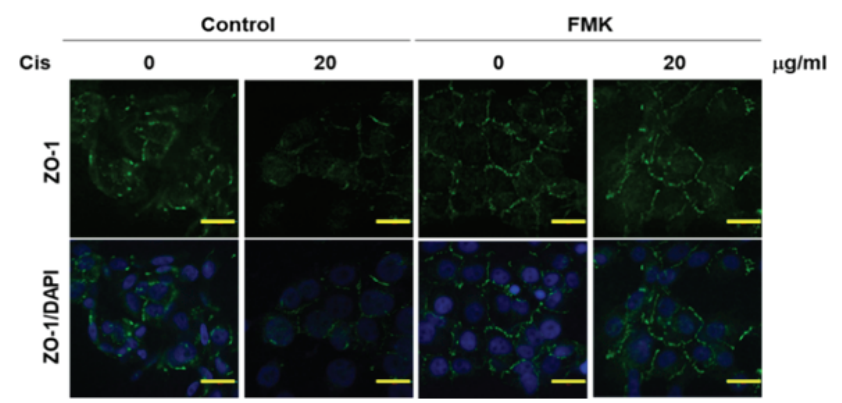

Fig. 4. Effect of p90RSK activation on EMT markers(A) E-cadherin; (B) N-cadherin; (C) Snail and (D) Twist mRNA level in MD A-MB-231 cells. MDA-MB-231 cells were transfected with si-Cont or si-RSK1 for $48 \mathrm{~h}$ followed by treatment with 0,10 , an $\mathrm{d} 20 \mathrm{ug} / \mathrm{ml}$ of Cis-DDP for $6 \mathrm{~h}$. Data are the mean \pm SEM of experiments in triplicate $(n=3)$. The data are presented as mean $\mathrm{s} \pm \mathrm{SEM}(\mathrm{n}=3) .{ }^{*} P<0.05,{ }^{* *} P<0.01$ compared with 0 sample; ${ }^{\#} P<0.5,{ }^{\# \#} P<0.01$ compared with each control. (E) MDA-MB-2 31 cells were treated with FMK for $3 \mathrm{~h}$ followed by treatment with $20 \mathrm{ug} / \mathrm{ml}$ of Cis-DDP for $6 \mathrm{~h}$. The ZO-1 expression in cell $\mathrm{s}$ was observed using a laser scanning confocal spectral microscope (K1-Fluo, Nanoscope systems). DAPI staining was used $t$ o determine the nuclei localization. Bars indicate $30 \mu \mathrm{m}$.

It has been known that EGF was related to breast cancer survival through Ras/Raf/MEK/ERK and PI3K/Akt signaling pathway $(4,20)$. In addition, EGF induced cell migration and anti-cancer drug resistance in breast cancer cells (18). In our study, Cis-DDP treatment increased mRNA expression of EGF in a dose dependent manner (Fig. 3). It suggests that Cis-DDP resistance is caused by EGFR activation. In addition, hEGF treatment induced phosphorylations of ERK1/2, p90RSK, and Akt (Fig. 3). Since ERK1/2 activity is important for regulating cancer cell proliferation (21) and ERK1/2 activation induces kinase activation in the C-terminal of p90RSK at the Serine 380 site (22), we investigated the role of p90RSK C-terminal kinase activation on TNBC signaling pathway. In the study, we found that FMK, a specific inhibitor of p90RSK C-terminal kinase activation, blocked EGF-mediated p90RSK activation.

Previous report demonstrated that an EMT transcription factor, Twist correlates with MAPK, which is one of the signaling pathways involved in the promotion of breast cancer cell invasion $(14,23)$. In addition, various EMT markers including Slug, Snail, and Twist have been reported to regulate the expression of tumor suppressor such as E-cadherin (16). We found that Cis-DDP-induced p90RSK activation was involved in EGF expression and EMT via upregulation of mesenchymal markers, such as N-cadherin, Snail, and Twist in Cis-DDP-stimulated MDA-MB-231 cells and downregulation of epithelial markers, such as E-cadherin and ZO-1 (Figs. 3 and 4). It suggests that blocking the C-terminal kinase activation of p90RSK is important for regulating Cis-DDP resistance of TNBC via suppressing EMT.

These discoveries reveal a new important mechanism in the research of Cis-DDP resistance in TNBC and that the regulation of C-terminal kinase activity of p90RSK can be a critical therapeutic target for increasing Cis-DDP sensitivity in patients with TNBC. 


\section{ACKNOWLEDGMENTS}

This research was supported by research fund of Chungnam National University.

\section{REFERENCES}

1) Jung J, Jang K, Ju JM, Lee E, Lee JW, Kim HJ, et al. Novel cancer gene variants and gene fusions of triple-negative breast cancers (TNBCS) reveal their molecular diversity conserved in the patient-derived xenograft (PDX) model. Cancer Lett 2018:428:127-38.

2) Feng F, Cheng P, Wang C, Wang Y, Wang W. Polyphyllin I and VII potentiate the chemosensitivity of A549/DDP cells to cisplatin by enhancing apoptosis, reversing EMT and suppressing the CIP2A/AKT/mTOR signaling axis. Oncol Lett 2019:18:5428-36.

3) Melissaridou S, Wiechec E, Magan M, Jain MV, Chung MK, Farnebo L, et al. The effect of 2D and 3D cell cultures on treatment response, EMT profile and stem cell features in head and neck cancer. Cancer Cell Int 2019;19:16.

4) Jung KH, Lee EJ, Park JW, Lee JH, Moon SH, Cho YS, et al. EGF receptor stimulation shifts breast cancer cell glucose metabolism toward glycolytic flux through PI3 kinase signaling. PLoS One 2019:14:e0221294.

5) Pedersen MH, Hood BL, Ehmsen S, Beck HC, Conrads TP, Bak M, et al. CYPOR is a novel and independent prognostic biomarker of recurrence-free survival in triple-negative breast cancer patients. Int J Cancer 2019;144:631-40.

6) Owonikoko TK, Dahlberg SE, Sica GL, Wagner LI, Wade JL 3rd, Srkalovic G, et al. Randomized Phase II Trial of Cisplatin and Etoposide in Combination With Veliparib or Placebo for Extensive-Stage Small-Cell Lung Cancer: ECOG-ACRIN 2511 Study. J Clin Oncol 2019;37:222-9.

7) Phelip JM, Edeline J, Blanc JF, Barbier E, Michel P, Bourgeois V, et al. Modified FOLFIRINOX versus CisGem first-line chemotherapy for locally advanced non resectable or metastatic biliary tract cancer (AMEBICA)-PRODIGE 38: Study protocol for a randomized controlled multicenter phase II/III study. Dig Liver Dis 2019;51:318-20.

8) Ludwik KA, Campbell JP, Li M, Li Y, Sandusky ZM, Pasic L, et al. Development of a RSK Inhibitor as a Novel Therapy for Triple-Negative Breast Cancer. Mol Cancer Ther 2016:15:2598-608.

9) Lin L, White SA, Hu K. Role of p90RSK in Kidney and Other Diseases. Int J Mol Sci 2019;20

10) Huynh DTN, Heo KS. Therapeutic targets for endothelial dysfunction in vascular diseases. Arch Pharm Res 2019; 42:848-61.

11) Chen S, Mackintosh C. Differential regulation of NHE1 phosphorylation and glucose uptake by inhibitors of the ERK pathway and p90RSK in 3T3-L1 adipocytes. Cell Signal 2009;21:1984-93.

12) Ikuta M, Kornienko M, Byrne N, Reid JC, Mizuarai S, Kotani $\mathrm{H}$, et al. Crystal structures of the N-terminal kinase domain of human RSK1 bound to three different ligands: Implications for the design of RSK1 specific inhibitors. Protein Sci 2007:16:2626-35 
13) Melhuish TA, Kowalczyk I, Manukyan A, Zhang $Y$, Shah A, Abounader R, et al. Myt1 and Myt1I transcription factors limit proliferation in GBM cells by repressing YAP1 expression. Biochim Biophys Acta Gene Regul Mech 2018;1861: 983-95.

14) Sheng W, Chen $C$, Dong M, Wang G, Zhou J, Song $H$, et al. Calreticulin promotes EGF-induced EMT in pancreatic cancer cells via Integrin/EGFR-ERK/MAPK signaling pathway. Cell Death Dis 2017:8:e3147.

15) Tas I, Han J, Park SY, Yang Y, Zhou R, Gamage CDB, et al. Physciosporin suppresses the proliferation, motility and tumourigenesis of colorectal cancer cells. Phytomedicine 2019;56:10-20.

16) Lin S, Zhang C, Liu F, Ma J, Jia F, Han Z, et al. Actinomycin V Inhibits Migration and Invasion via Suppressing Snail/Slug-Mediated Epithelial-Mesenchymal Transition Progression in Human Breast Cancer MDA-MB-231 Cells In Vitro. Mar Drugs 2019;17.

17) Heo KS, Le NT, Cushman HJ, Giancursio CJ, Chang E, Woo CH, et al. Disturbed flow-activated p90RSK kinase accelerates atherosclerosis by inhibiting SENP2 function. J Clin Invest 2015:125:1299-310.

18) Tang T, Zhu Q, Li X, Zhu G, Deng S, Wang Y, et al. Protease Nexin I is a feedback regulator of EGF/PKC/MAPK/EGR1 signaling in breast cancer cells metastasis and stemness. Cell Death Dis 2019;10:649.

19) Li L, Su N, Zhou T, Zheng D, Wang Z, Chen H, et al. Mixed lineage kinase ZAK promotes epithelial-mesenchymal transition in cancer progression. Cell Death Dis 2018;9:143.

20) Suen KM, Lin CC, Seiler C, George R, Poncet-Montange G, Biter AB, et al. Phosphorylation of threonine residues on Shc promotes ligand binding and mediates crosstalk between MAPK and Akt pathways in breast cancer cells. Int J Biochem Cell Biol 2018;94:89-97.

21) Lim W, Yang C, Park S, Bazer FW, Song G. Inhibitory Effects of Quercetin on Progression of Human Choriocarcinoma Cells Are Mediated Through PI3K/AKT and MAPK Signal Transduction Cascades. J Cell Physiol 2017;232:1428-40.

22) Singh MV, Kotla S, Le NT, Ae Ko K, Heo KS, Wang Y, et al. Senescent Phenotype Induced by p90RSK-NRF2 Signaling Sensitizes Monocytes and Macrophages to Oxidative Stress in HIV-Positive Individuals. Circulation 2019;139:1199-216.

23) Xu M, Wang S, Wang Y, Wu H, Frank JA, Zhang Z, et al. Role of p38gamma MAPK in regulation of EMT and cancer stem cells. Biochim Biophys Acta Mol Basis Dis 2018;1864:3605-17. 